Original Research Paper

\title{
Bifurcation and Controlling Chaos in a Discrete-Time Biological System
}

\author{
Guo Feng \\ School of Data and Computer Science, Shandong Women's University, Jinan, China 250300
}

Article history

Received: 11-06-2020

Revised: 22-06-2020

Accepted: 06-07-2020

Email: tk524924@163.com

\begin{abstract}
The dynamics of a discrete-time food chain model with strong pressure on preys is investigated. The types of equilibria of the system are analyzed using stability theory and bifurcation theory. The route to chaos via Neimark-Sacker bifurcation followed by period-doubling bifurcations of invariant curves is found for some parameter values through numerical simulation. Moreover, the chaos is controlled on the stable periodic period1 orbit by the improvement of OGY method. It is shown that the number of iterations used to control chaotic motion on a stable periodic orbit is difference, when the selected regulator poles are different. Numerical simulations are presented to illustrate our results based on the theoretical analysis and show the effect of the control method.
\end{abstract}

Keywords: Food Chain Model, Neimark-Sacker Bifurcation, Chaos Control, OGY Method

\section{Introduction}

The dynamics in species was studied in the late 1990's (Costantino et al., 1997; Dennis et al., 2001). More and more researchers have studied the dynamic behavior of discrete ecosystems. These ecological systems have complex dynamics behaviors and many valuable results have been obtained (May, 1976; Hastings and Powell, 1991). The dynamics of three species discrete-time food chain models was investigated (Elsadany, 2012; Zhang and Zhao, 2009; Alba-Pérez and Macias-Diaz, 2019; Asheghi, 2014) studied discrete food chain systems with a functional response of Beddington-DeAngelis.

Ott et al. (1990) proposed a method to control chaos. The early research idea is to use the existing dynamics control strategies and destroy the conditions for the occurrence of chaotic motion. Pyragas (1995; Shinbrot et al., 1993; Kacarev and Parlitz, 1996) have put forward different improvement measures according to various situations and further developed the OGY method, which has laid a good foundation for the application of chaos.

Guo et al. (2014a; 2014b) studied the chaos control of the Lauwerier mapping:

$\left\{\begin{array}{c}x_{n+1}=b x_{n}\left(1-2 y_{n}\right)+y_{n} \\ y_{n+1}=a y_{n}\left(1-y_{n}\right)\end{array}\right.$

and the Lozi mapping:

$$
\left\{\begin{array}{c}
x_{n+1}=1-a\left|x_{n}\right|+y_{n} \\
y_{n+1}=b x_{n}
\end{array}\right.
$$

By the OGY method. The chaotic motions of Equation (1 and 2) are both controlled on periodic-1 and periodic-2 orbits. Feng et al. (2018) studied the chaos control of two degree of freedom collision vibration system through OGY method. A four-dimensional discontinuous system is studied. The chaotic motion is controlled on periodic- 1 and periodic- 2 orbits.

Recently, the chaos control in biological systems has been studied. Many chaos control methods can be applied to population dynamics and play nontrivial evolutionary roles in ecology (Bešo et al., 2020; 2019; Din, 2017; Din et al., 2018; Din, 2018a; 2018b; Kapçak et al., 2013).

The three-dimensional model explored in this paper introduces a top predator species that consumes the predator and interferes in the growth of the preys. The system has a chaotic attractor when $a=2.1, b=3.36, c=$ 7.4. The chaotic dynamics of this system is investigated and the chaos is controlled. The unstable point is controlled on the period-1orbit. It is shown that the control times depend on the adjustment values.

The aim of this paper is to control the chaotic motion of a three-dimensional food model use the OGY method. The process of realizing the control is only a small continuous disturbance to the parameters. The dynamic properties of the original system are kept. Other control methods change the balance point of the system and the fixed point of the control is no longer the original system. This method is not studied in the previous literature. 
In biology or ecology, the chaotic behavior of this model shows the relations of the different species, including the number population, birth rate and survival rate, whether they can survive in a balanced state or makes the population develop in disorder or chaos. This research can provide theoretical basis and help for the research in biology or ecology.

This paper is organized as follows. In section 2, we discuss the existence and stability of fixed points of the system (3). We present numerical simulations, which exhibit the complex dynamical behaviors such as NeimarkSacker bifurcation and chaotic sets. In section 3 , chaos is controlled to an unstable fixed point using the OGY control method. A brief discussion is given in section 4 .

\section{The Equilibria and the Dynamics Analysis of a Three-Species Food Chain Model}

We consider a three-species food chain model described by the nonlinear difference equations:

$F:\left\{\begin{array}{c}x_{n+1}=a x_{n}\left(1-x_{n}-y_{n}-z_{n}\right) \\ y_{n+1}=b y_{n}\left(x_{n}-z_{n}\right) \\ z_{n+1}=c y_{n} z_{n}\end{array}\right.$

This model describes the insects group of three fully different insects. The parameters $a, b, c$ are positive. In the absence of predation, preys grow logistically with an intrinsic reproduction rate $a$. However, preys' reproduction is decreased by the action of predation from both predators $y$ and $z$. Parameter $b$ is the growth rate of predators $y$, which is proportional to the consumption of preys. $c$ is the growth rate of predators $z$ due to the consumption of species $y$. Notice that predator $z$ also predates (interferes) on $x$, but it is assumed that the increase in reproduction of the top predator $z$ is mainly given by the consumption of species $y$.

In order to find out the equilibria of this system and study its dynamic properties. Equation (3) is written:

$\left\{\begin{array}{l}f(x, y, z)=x \\ g(x, y, z)=y \\ h(x, y, z)=z\end{array}\right.$

where, $f(x, y, z)=\operatorname{ax}(1-x-y-z), g(x, y, z)=b y(x-z), h(x, y, z)=$ $c y z$. The Equation (3) has four equilibria:

$$
\begin{aligned}
& E_{1}=(0,0,0) \\
& E_{2}=\left(\frac{a-1}{a}, 0,0\right) \\
& E_{3}=\left(\frac{1}{b}, 1-\frac{1}{a}-\frac{1}{b}, 0\right) \\
& E_{4}=\left(\frac{1}{2}\left(1-\frac{1}{a}+\frac{1}{b}-\frac{1}{c}\right), \frac{1}{c}, \frac{1}{2}\left(1-\frac{1}{a}-\frac{1}{b}-\frac{1}{c}\right)\right)
\end{aligned}
$$

Assume that, $E_{i}\left(x_{*}, y_{*}, z_{*}\right)(i=1,2,3,4)$ is positive and denote equilibrium point of the Equation (3).

To carry out linear stability analysis, the Taylor series expansion of Equation (3) may be written as:

$$
\left(\begin{array}{c}
x_{t+1} \\
y_{t+1} \\
z_{t+1}
\end{array}\right)=\left(\begin{array}{lll}
\frac{\partial f}{\partial x} & \frac{\partial f}{\partial y} & \frac{\partial f}{\partial z} \\
\frac{\partial g}{\partial x} & \frac{\partial g}{\partial y} & \frac{\partial g}{\partial z} \\
\frac{\partial h}{\partial x} & \frac{\partial h}{\partial y} & \frac{\partial h}{\partial z}
\end{array}\right)_{\left(x_{*}, y_{*}, z_{*}\right)}\left(\begin{array}{c}
x_{t} \\
y_{t} \\
z_{t}
\end{array}\right)
$$

Using Equation (4) and (5), one can obtain the following:

$$
\begin{aligned}
& \left.\left.\frac{\partial f}{\partial x}\right|_{\left(x_{*}, y_{*}, z_{*}\right)}=a\left(1-2 x_{*}-y_{*}-z_{*}\right),\left.\frac{\partial f}{\partial y}\right|_{\left(x_{*}, y_{*}, z_{*}\right)}=-a x_{*},\left.\frac{\partial f}{\partial z}\right|_{\left(x_{*}, y_{*}, z_{*}\right)}=b x_{*},\left.\frac{\partial g}{\partial y}\right|_{\left(x_{*}, y_{*}, z_{*}\right)}=b\left(x_{*}-z_{*}\right),\left.\frac{\partial g}{\partial z}\right|_{\left(x_{*}, y_{*}, z_{*}\right)}=b z_{*}\right) \\
& =-\left.b y_{*} \frac{\partial h}{\partial x}\right|_{\left(x_{*}, y_{*}, z_{*}\right)}=0,\left.\frac{\partial h}{\partial y}\right|_{\left(x_{*}, y_{*}, z_{*}\right)}=c z_{*},\left.\frac{\partial h}{\partial z}\right|_{\left(x_{*}, y_{*}, z_{*}\right)}=c y_{*}
\end{aligned}
$$

Consider the matrix:

$$
A=\left(\begin{array}{lll}
P_{11} & P_{12} & P_{13} \\
P_{21} & P_{22} & P_{23} \\
P_{31} & P_{32} & P_{33}
\end{array}\right)
$$

where:

$$
\begin{aligned}
& P_{11}=\left.\frac{\partial f}{\partial x}\right|_{\left(x_{*}, y_{*}, z_{*}\right)}, P_{12}=\left.\frac{\partial f}{\partial y}\right|_{\left(x_{*}, y_{*}, z_{*}\right)}, P_{13}=\left.\frac{\partial f}{\partial z}\right|_{\left(x_{*}, y_{*}, z_{*}\right)} \\
& P_{21}=\left.\frac{\partial g}{\partial x}\right|_{\left(x_{*}, y_{*}, z_{*}\right)}, P_{22}=\left.\frac{\partial g}{\partial y}\right|_{\left(x_{*}, y_{*}, z_{*}\right)}, P_{23}=\left.\frac{\partial g}{\partial z}\right|_{\left(x_{*}, y_{*}, z_{*}\right)} \\
& P_{31}=\left.\frac{\partial h}{\partial x}\right|_{\left(x_{*}, y_{*}, z_{*}\right)}, P_{32}=\left.\frac{\partial h}{\partial y}\right|_{\left(x_{*}, y_{*}, z_{*}\right)}, P_{33}=\left.\frac{\partial h}{\partial z}\right|_{\left(x_{*}, y_{*}, z_{*}\right)}
\end{aligned}
$$

The characteristic equation is:

$$
\operatorname{det}\left(\begin{array}{ccc}
P_{11}-\lambda & P_{12} & P_{13} \\
P_{21} & P_{22}-\lambda & P_{23} \\
P_{31} & P_{32} & P_{33}-\lambda
\end{array}\right)=0
$$

Which may be rewritten in the form:

$$
\lambda^{3}+c_{1} \lambda^{2}+c_{2} \lambda+c_{3}=0
$$

where: 
$c_{1}=-\left(P_{11}+P_{22}+P_{33}\right)$

$c_{2}=P_{11} P_{22}-P_{12} P_{21}+P_{11} P_{33}-P_{13} P_{31}+P_{22} P_{33}-P_{23} P_{32}$

$c_{3}=-P_{11} P_{22} P_{33}+P_{11} P_{23} P_{32}+P_{12} P_{21} P_{33}-P_{12} P_{23} P_{31}-P_{13} P_{21} P_{32}+P_{13} P_{22} P_{31}$

For the parameter values $a=2.1, b=3.36, c=7.4$, the eigenvalues can be obtained for the four equilibria, so we can judge the type of each equilibrium point:

(i) The eigenvalues are $\lambda_{11}=a, \lambda_{12}=\lambda_{13}=0$ at $E_{1}$, so $E_{1}$ is a saddle point

(ii) The eigenvalues are $\lambda_{21}=-0.9, \lambda_{22}=1.76, \lambda_{23}=0$ at $E_{2}$, so $E_{2}$ is a saddle point

(iii) The eigenvalues are $\lambda_{31}=1.674, \lambda_{32}=$ $0.6651+0.6143 i, \lambda_{33}=0.6651-0.6143 i$ at $E_{3}$, so $E_{3}$ is an unstable spiral-sink node source

(iv) The eigenvalues are $\lambda_{41}=0.3923, \lambda_{42}=0.9329$ $0.6313 i, \lambda 43=0.9329-0.6313 i$ at $E_{4}$, so $E_{4}$ is a locally asymptotically stable sink of spiral node

Alsedà et al. (2012) made a detailed analysis of the equilibria of the system. Different parameter ranges were given to make the four equilibria $\left(E_{1}, E_{2}, E_{3}, E_{4}\right)$ of the system stable or unstable. Let us explore the dynamics of the system focusing on the strength of predation,

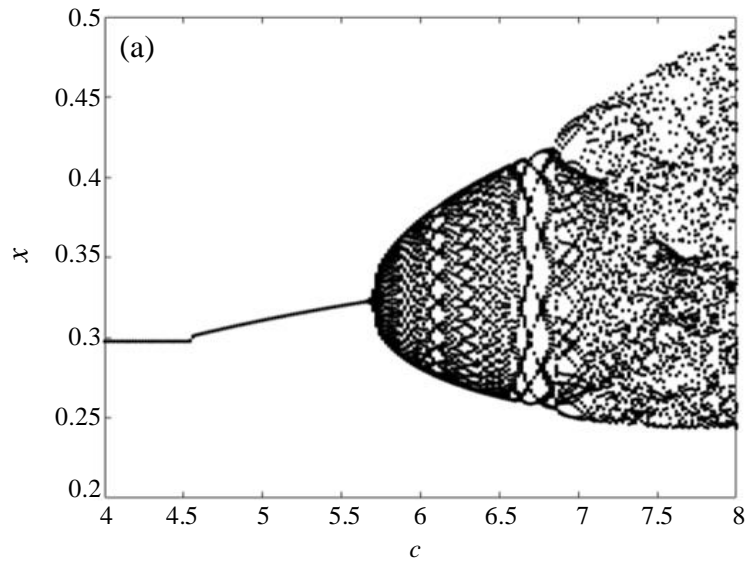

parametrized by constant $c$. Without loss of generality, we take $a=2.1, b=3.36$ and assume that $c$ changes from 5.5 to 7.4. Under the assumption of such parameter values, the dynamics behavior of this system is mainly studied by numerical analysis.

The bifurcation diagrams of Equation (3) is shown in Fig. 1a to c for each species $x, y$ and $z$ with $a=2.1, b=$ 3.36 , as the parameter $c$ changes from 4 to 8 . We now discuss the salient features of the bifurcation diagram. The bifurcation diagrams computed in Fig. 1 seem to indicate that after a Neimark-Sacker bifurcation, the new invariant curves undergo period-doublings.

When the reproduction rate $c$ changes between 4 and 8, Equation (3) generates complicated features. The equilibria $E_{4}$ is a positive fixed point that is asymptotically stable for $5<c<5.67$. The phase portraits of various $c$ corresponding to Fig. 1a are plotted in Fig. $2 \mathrm{a}$ and at $c=5.67$, a Neimark-Sacker bifurcation appears. When the parameter $c$ changes from 5.67 to 6.58 , there is one invariant circle in the system. With the change of parameters for $6.58<c<7.15$, a perioddoubling bifurcation appears and there are two invariant circles in the system.
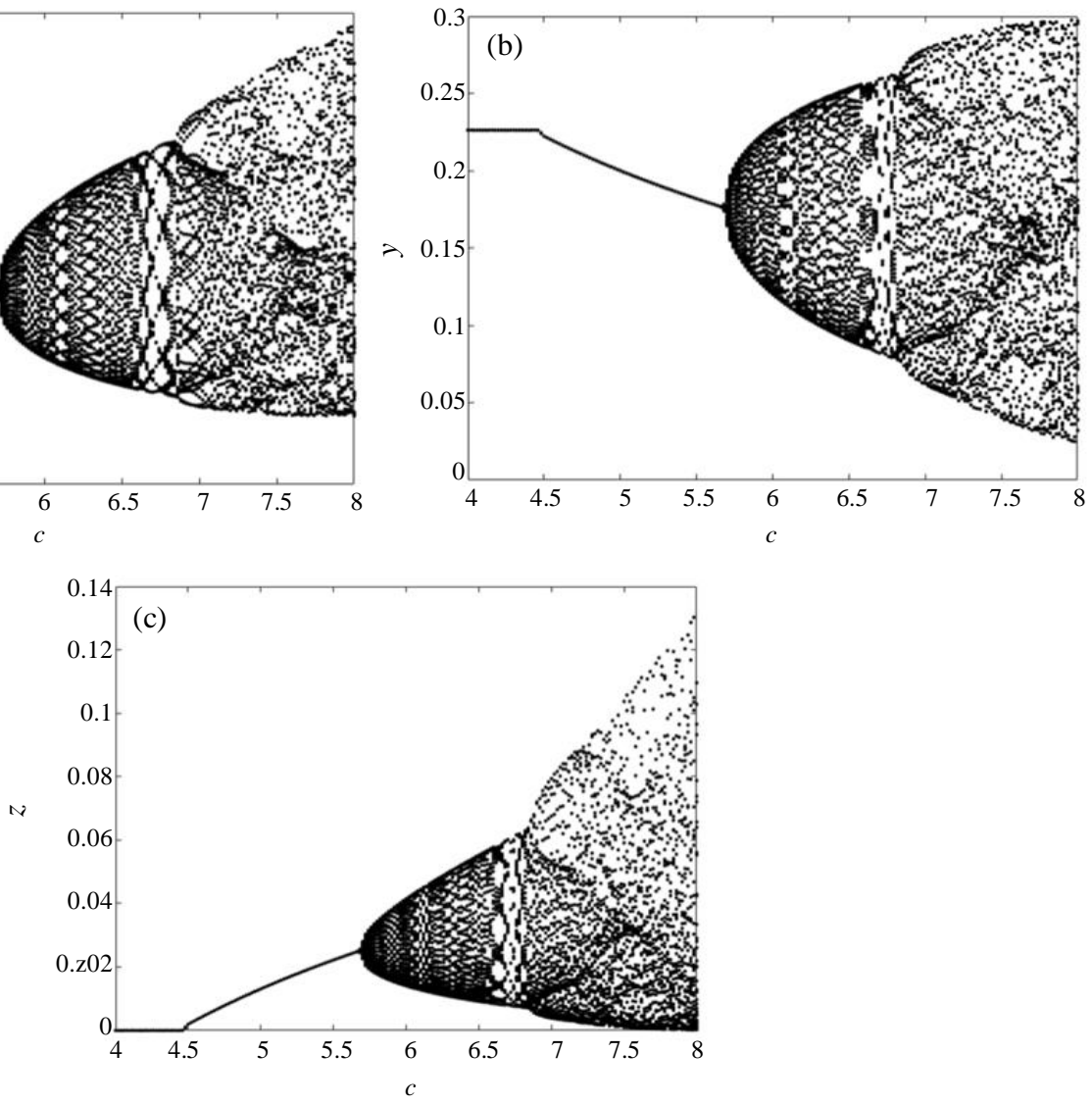

Fig. 1: The bifurcation diagrams of Equation (3) for each species $x, y$ and $\mathrm{z}$ with $a=2.1, b=3.36$, as the parameter c changes from 4 to 8 . 


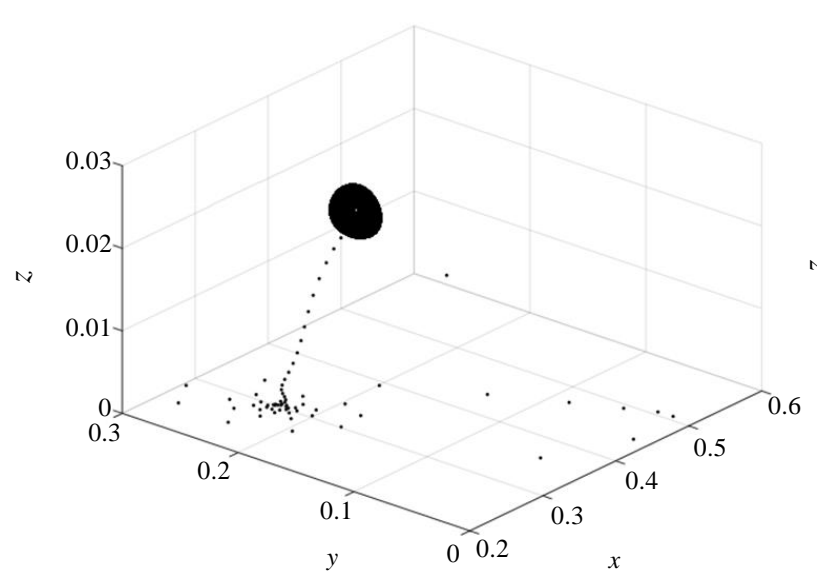

(a)

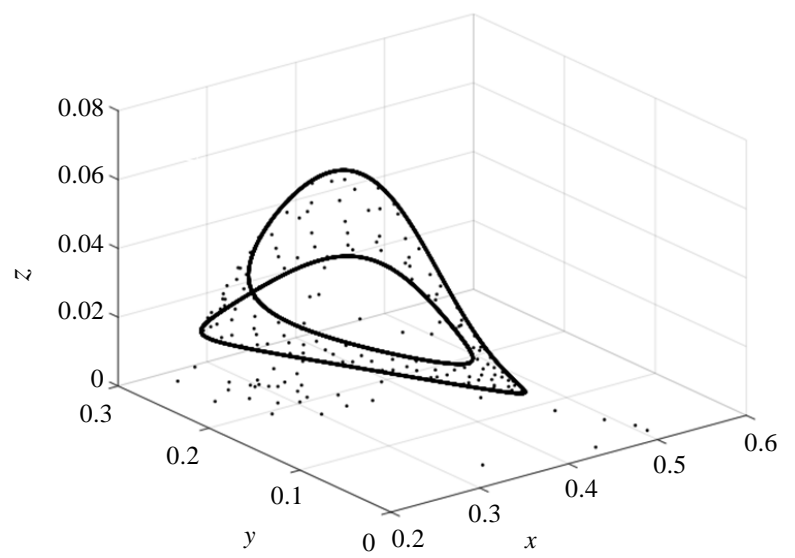

(c)

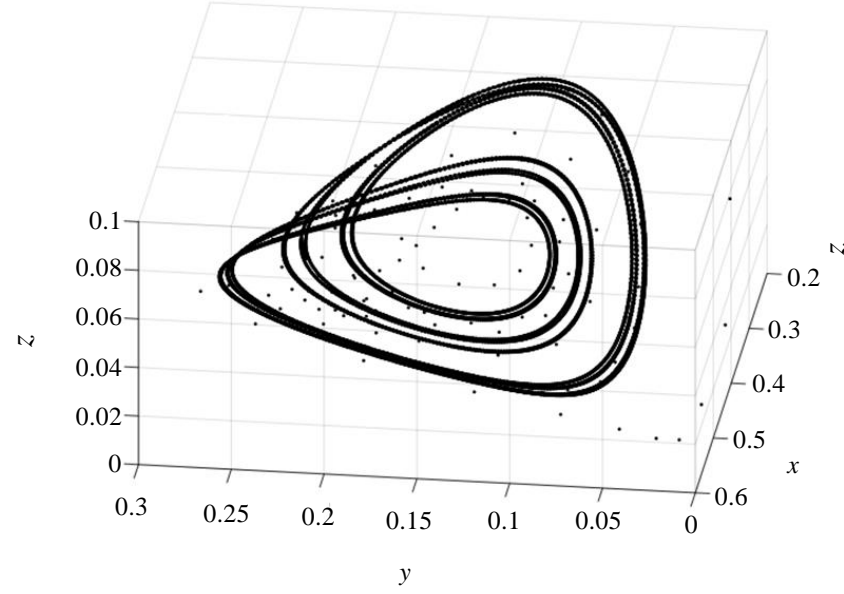

(e)

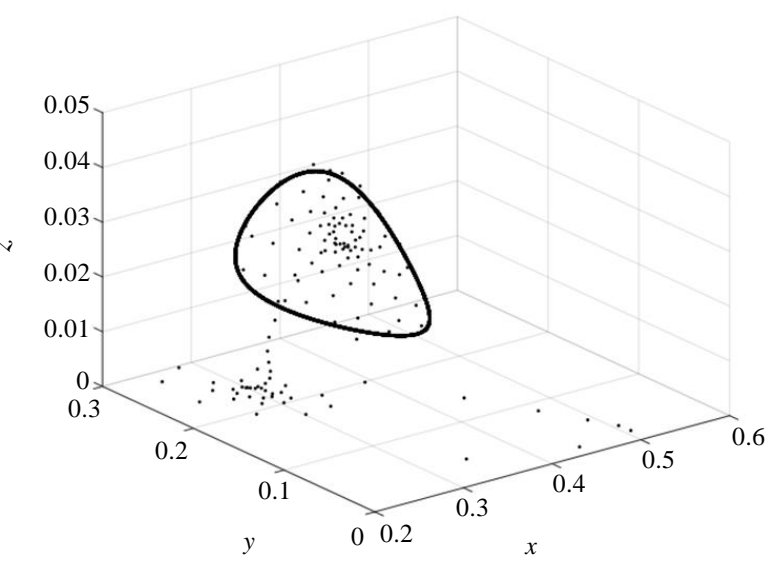

(b)

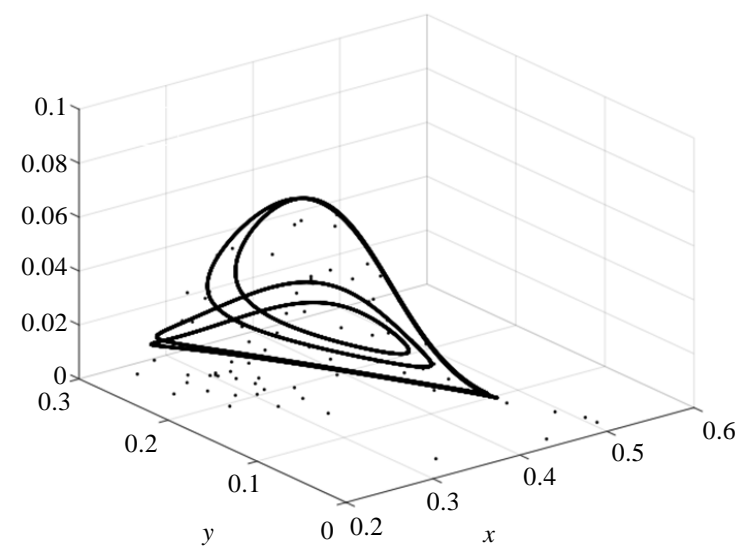

(d)

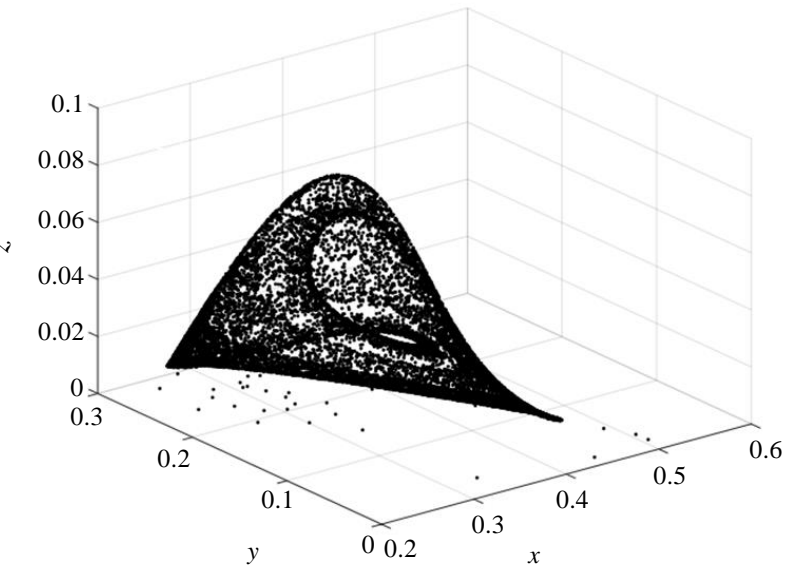

(f)

Fig. 2: The phase diagrams of Equation (3) with $a=2.1, b=3.36$, as the different parameters $c$ : ((a) $5<c<5.67$, (b) $5.67<c<6.58$, (c) $6.58<c<7.15$, (d) $7.15<c<7.25$, (e) $7.25<c<7.4$, (f) $c=7.4$ )) 
With the increase of parameter $c$ changing from 7.15 to 7.25 , the system appears a period-doubling bifurcation, there are four invariant circles. With the increase of parameter $c$ changing from 7.25 to 7.4 , the system appears a period-doubling bifurcation, there are eight invariant circles. When the parameter $c>7.4$, the system appears chaotic dynamic behavior. The phase portraits of various $c$ corresponding to Fig. 1a are plsotted in Fig. $2 \mathrm{~b}$ to $2 \mathrm{f}$.

When the parameter $c$ passes through the range (7.28, 7.29) and (7.37, 7.29) Fig. 1a, the Equation (3) goes through a quasi-periodic region (wide periodic windows and frequency locking which appear as a collapse of the invariant circle to a periodic orbit). Then, chaos appears after period-doubling bifurcation.

\section{The Chaos Control of the Three-Species Food Chain Model}

As shown in Fig. 3, for $a=2.1, b=3.36$, when $c=$ 7.4, the dynamics behavior of the system is chaotic. Moreover, there exists a chaotic attractor which is the closure of the unstable manifolds of the saddle points and there are an infinite number of unstable periodic orbits in the chaotic attractor. In particular, there are unstable period1 orbits embedding in the chaotic attractor.

The chaotic motion of the three-species food chain model is controlled to the stable periodic period- 1 orbit through the improvement of OGY method. Control parameter $c$ is perturbed slightly depending times. When the unstable point wanders to the neighborhood of the periodic-1 orbit, the control parameter is perturbed.

Write the system (?) in the following form:

$W_{i+1}=F\left(W_{i}, c\right), W_{i} \in R^{3}, c \in R$

where, $F$ is sufficiently smooth, $c$ is an externally adjustable real parameter. That is required $|c-\bar{c}|<\delta$ at some time and $\bar{c}$ is a rated value. Let the control parameter $c$ be a variable parameter near the rated value $\bar{c}=7.4$. For $a$ $=2.1, b=3.36$, by the Equation (4), the equilibrium point $E_{4}$ is $E_{i}\left(x_{*}, y_{*}, z_{*}\right)=(0.3474,0.1266,0.0498)$.

Now the aim is to change the parameter such that the chaotic attractor involves almost all of the initial conditions, so that the dynamics behavior of the system converges to the desired periodic orbit in the attractor. Through the OGY method, due to the ergodicity of the chaotic motions, when the state orbit enter the vicinity of the unstable periodic orbit to be stabilized, a feedback control law is applied to control the trajectory to move to the desired unstable periodic orbit.

Denoted by $W_{*}(\bar{c})$ the unstable fixed point $E_{4}$. By the first-order Taylor expansion, Equation (13) can be written as:
$W_{i+1}-W_{*}(\bar{c})=\mathrm{A}\left(W_{i}-W_{*}(\bar{c})\right)+\mathrm{B}(c-\bar{c})$

Find out the matrices $A$ and $B$ at $W=W_{*}(\bar{c})$ and $c=\bar{c}$, where $A$ is the partial derivative matrix of $F(w, c)$ to $w$ and $w$ is the variable set of $(x, y, z), A=D_{w} F(w, c)$ :

$\mathrm{A}=\left(\begin{array}{ccc}a(1-2 x-y-z) & -a x & -a x \\ b y & b(x-z) & -b y \\ 0 & c z & c y\end{array}\right)$

and $\mathrm{B}$ is the partial derivative matrix of $F(w, a)$ to $a$, $B=D c F(w, c)$ :

$\mathrm{B}=\left(\begin{array}{c}x(1-x-y-z) \\ 0 \\ 0\end{array}\right)$

Bring the equilibrium point $E_{4}\left(x_{*}, y_{*}, z^{*}\right)=(0.3474$, $0.1266,0.0498)$ into the matrixes $A$ and $B$ :

$\mathrm{A}=\left(\begin{array}{ccc}0.2705 & -0.7295 & -0.7295 \\ 0.4254 & 1 & -0.4254 \\ 0 & 0.3884 & 0.9875\end{array}\right) \mathrm{B}=\left(\begin{array}{c}0.1654 \\ 0 \\ 0\end{array}\right)$

The time dependent control parameter $c$ is in the form of a linear function with respect to the variable:

$c-\bar{c}=-\mathrm{K}^{\mathrm{T}}\left(W_{i}-W_{*}(\bar{c})\right)$

Substituting Equation (18) into Equation (14):

$W_{i+1}-W_{*}(\bar{c})=\left(\mathrm{A}-\mathrm{BK}^{\mathrm{T}}\right)\left(W_{i}-W_{*}(\bar{c})\right)$

So as long as the matrix $A-B K^{T}$ is asymptotically stable, that is, if the modulus of its eigenvalues are less than 1 , the equilibrium point $W_{*}(\bar{c})$ is stable. The following key question is how to determine the matrix $K^{T}$, which can stabilize the chaotic motion at a stable periodic point. The pole assignment is solved according (Ogata, 1990). The matrix $C_{3 \times 3}$ is controllable matrix:

$\mathrm{C}=\left(\begin{array}{lll}\mathrm{B} & \mathrm{AB} & \mathrm{A}^{2} \mathrm{~B}\end{array}\right)=\left(\begin{array}{ccc}0.1654 & 0.0447 & -0.0392 \\ 0 & 0.0704 & 0.0894 \\ 0 & 0 & 0.0273\end{array}\right)$ 
The solution of pole assignment is given by KT $=\left(\alpha_{3}\right.$ - $\alpha_{3}-\alpha_{2}-\alpha_{2}-\alpha_{1}-a_{1}$ ), where $T=C Q$ and $\mathrm{Q}$ is a matrix of order 3 :

$\mathrm{Q}=\left(\begin{array}{ccc}a_{2} & a_{1} & 1 \\ a_{1} & 1 & 0 \\ 1 & 0 & 0\end{array}\right)$

$a_{i}(i=1,2,3)$ are the coefficients of the characteristic polynomial of the matrix $A$, that is:

$\operatorname{det}(\lambda \mathrm{I}-\mathrm{A})=\lambda^{3}+a_{1} \lambda^{2}+a_{2} \lambda+a_{3}$

Substituting the matrix $A$ into Equation (22):

$\operatorname{det}(\lambda \mathrm{I}-\mathrm{A})=\operatorname{det}\left(\begin{array}{ccc}\lambda-0.2705 & 0.7295 & 0.7295 \\ -0.4254 & \lambda-1 & 0.4254 \\ 0 & -0.3884 & \lambda-0.9875\end{array}\right)$

$=-\lambda^{3}-2.2580 \lambda^{2}+2.001 \lambda-0.4977$

yields, $a_{1}=-2.2580, a_{2}=2.001, a_{3}=-0.4977$

Assume that $\alpha_{1}, \alpha_{2}, \alpha_{3}$ are the coefficients of the characteristic polynomial $\operatorname{det}\left(A-B K^{T}\right)$, that is:

$\operatorname{det}\left(s \mathrm{I}-\left(\mathrm{A}-\mathrm{BK}^{\mathrm{T}}\right)\right)=s^{3}+\alpha_{1} s^{2}+\alpha_{2} s+\alpha_{3}$

$\mathrm{T}=\mathrm{CQ}=\left(\begin{array}{ccc}0.1654 & 0.0447 & -0.0392 \\ 0 & 0.0704 & 0.0894 \\ 0 & 0 & 0.0273\end{array}\right)\left(\begin{array}{ccc}2.001 & -2.2580 & 1 \\ -2.2580 & 1 & 0 \\ 1 & 0 & 0\end{array}\right)(25)$

$=\left(\begin{array}{ccc}0.1908 & -0.3288 & 0.1654 \\ -0.0696 & 0.0704 & 0 \\ 0.0273 & 0 & 0\end{array}\right)$

$\mathrm{T}^{-1}=\left(\begin{array}{ccc}0 & 0 & 36.6300 \\ 0 & 14.2045 & 36.1946 \\ 6.0459 & 28.2350 & 29.6833\end{array}\right)$

The eigenvalues of the matrix $\mathrm{A}$ at the equilibrium point $\left(x_{*}, y_{*}, z^{*}\right)=(0.3474,0.1266,0.0498)$ are:

$$
\begin{aligned}
& \lambda_{s}=0.3923, \\
& \lambda_{1 u}=0.9329+0.6313 \mathrm{i}, \\
& \lambda_{2 u}=0.9329-0.6313 \mathrm{i}
\end{aligned}
$$

Assume $\alpha_{1}, \alpha_{2}, \alpha_{3}$ are the coefficients of the characteristic polynomial of the matrix $A-B K^{T}$. The assumed characteristic roots $\mu_{1}, \mu_{2}, \mu_{3}$ are called the adjustment values, that is: $\operatorname{det}\left(s \mathrm{I}-\left(\mathrm{A}-\mathrm{BK}^{\mathrm{T}}\right)\right)=s^{3}-\left(\mu_{1}+\mu_{2}+\mu_{3}\right) s^{2}$
$+\left(\mu_{1} \mu_{2}+\mu_{2} \mu_{3}+\mu_{1} \mu_{3}\right) s-\mu_{1} \mu_{2} \mu_{3}$

The relation between the roots and the coefficients is given by:

$\alpha_{1}=-\left(\mu_{1}+\mu_{2}+\mu_{3}\right), \alpha_{2}=\mu_{1} \mu_{2}+\mu_{2} \mu_{3}+\mu_{1} \mu_{3}, \alpha_{3}=-\mu_{1} \mu_{2} \mu_{3}$

From Ogata (1990) and Equation (13), the matrix $K^{T}$ is not unique. As long as the matrix $K^{T}$ is obtained with the values $\alpha_{1}, \alpha_{2}$ and $\alpha_{3}$, it can make the matrix $A-B K^{T}$ be asymptotically stable, the modulus of their eigenvalues are less than 1 , so we can take $\left(\alpha_{1}, \alpha_{2}, \alpha_{3}\right)$ $=\left(-\left|\lambda_{s}\right|,-\lambda_{s} \mid, 0\right)$ and obtain:

$$
\begin{aligned}
& \mathrm{K}^{\mathrm{T}}=\left(0-a_{3}-\left|\lambda_{s}\right|-a_{2}-\left|\lambda_{s}\right|-a_{1}\right) \mathrm{T}^{-1} \\
& =\left(\begin{array}{lll}
0.4977 & -2.3933 & 1.8657
\end{array}\right)\left(\begin{array}{ccc}
0 & 0 & 36.6300 \\
0 & 14.2045 & 36.1946 \\
6.0459 & 28.2350 & 29.6833
\end{array}\right) \\
& =\left(\begin{array}{lll}
11.2799 & 18.6824 & -13.0138
\end{array}\right)
\end{aligned}
$$

when, $K^{T}$ is found, $\left|\mathrm{K}^{\mathrm{T}}\left(W_{i}-W_{*}(\bar{a})\right)\right|<\delta$ is obtained by $|c-\bar{c}|<\delta$ and the Equation (14). There is a region whose width is:

$$
2 \delta /\left|\mathrm{K}^{\mathrm{T}}\right|
$$

when, $W_{i}$ in this region, the parameter is controlled, otherwise the parameter is not controlled. The control rate is given by the following formula:

$c-\bar{c}=-\mathrm{K}^{\mathrm{T}}\left(W_{i}-W_{*}(\bar{c})\right), \times u\left(\delta-\left|\mathrm{K}^{\mathrm{T}}\left(Z_{i}-Z_{*}(\bar{c})\right)\right|\right)$,

where:

$u(\alpha)=\left\{\begin{array}{l}0, \alpha<0 \\ 1, \alpha>0\end{array}\right.$

As shown in Fig. 4, when $\alpha_{1}=0.3923, \alpha_{2}=$ $0.3923, \alpha_{3}=0$, the chaotic motion can be controlled to the period- 1 orbit at $n=820 \mathrm{Fig}$. 4a to $4 \mathrm{c}$ ). When $\alpha_{1}=0.256, \alpha_{2}=0.465, \alpha_{3}=0.210$, the chaotic motion can be controlled on the period-1 orbit at $n=2400$ Fig. $4 \mathrm{~d}$ to $4 \mathrm{f}$. When the values of $\alpha_{1}, \alpha_{2}, \alpha_{3}$ are different from the previous ones, the number of the Equation (3) iterations is different to control chaos. 


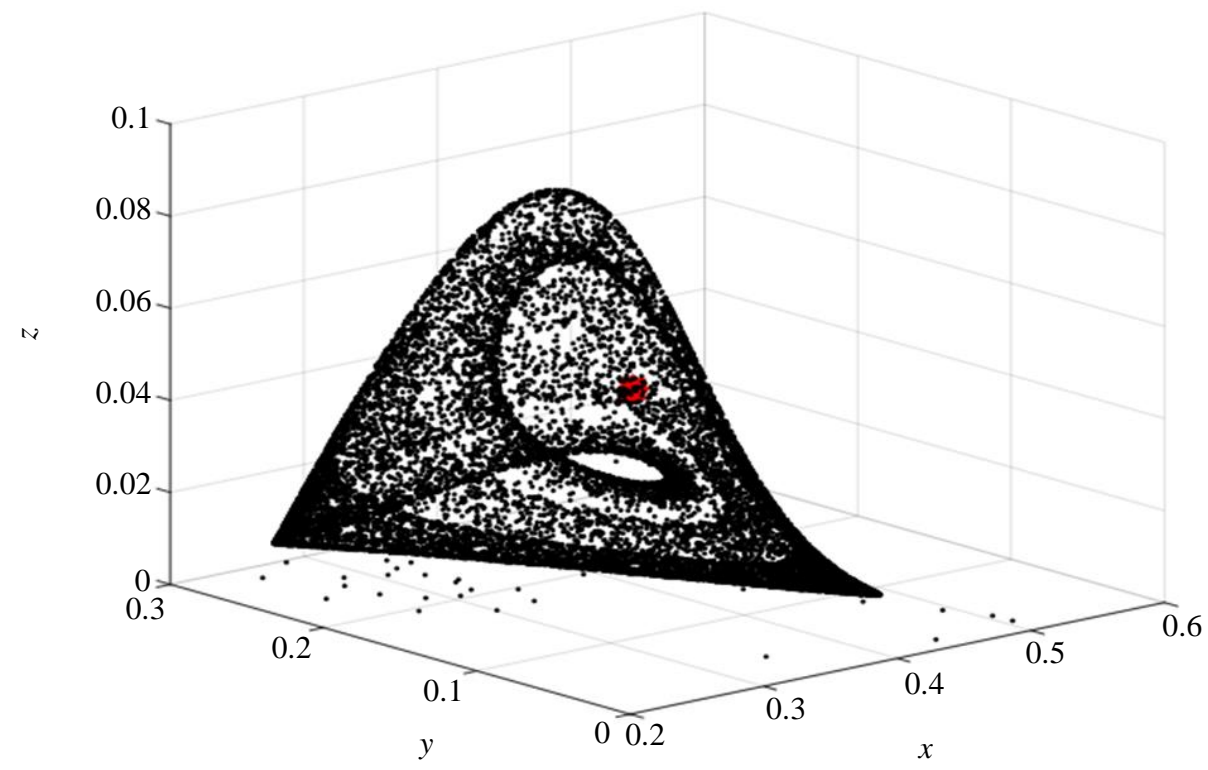

Fig. 3: The chaotic attractor of food chain model with an unstable fixed point $(a=2.1, b=3.36, c=7.4)$
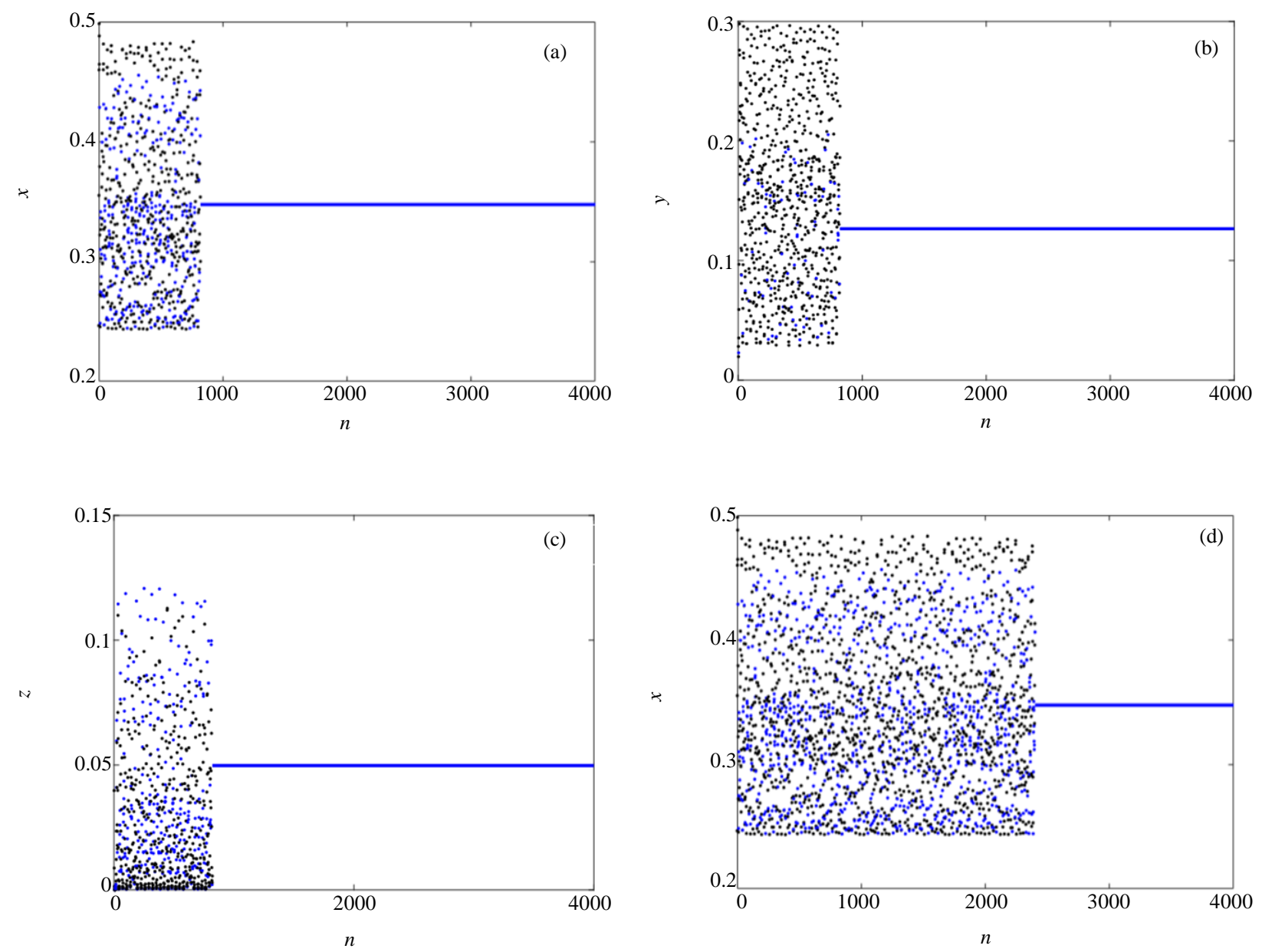

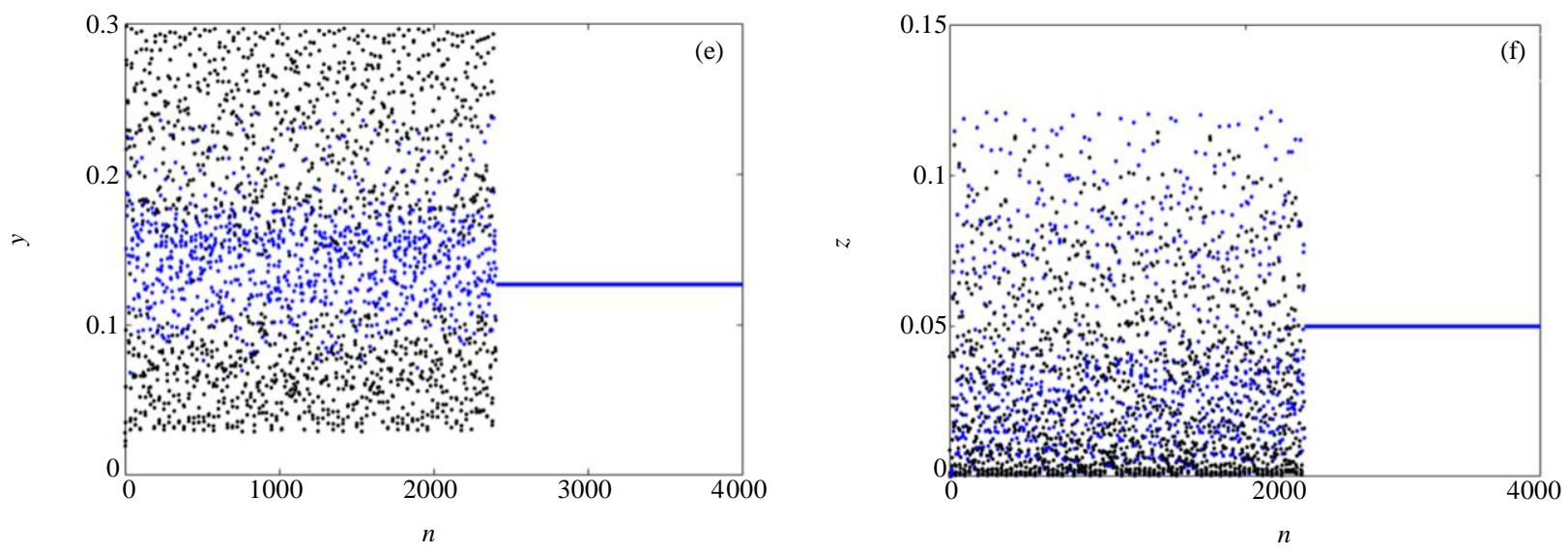

Fig. 4: Control of period-1 of food chain model

\section{Conclusion}

The dynamics of a discrete-time food chain model with strong pressure on preys is investigated. The types of equilibria of the system are analyzed. The chaos is achieved via a Neimark-Sacker bifurcation followed by period-doubling bifurcations of invariant curves for some parameter values. The chaotic motion is controlled to the stable periodic period-1 orbit using the improvement of OGY method. It is shown that the number of iterations used to control chaotic motion on a stable periodic orbit is difference, when the selected regulator poles are different. The process of realizing the control is only a small continuous disturbance to the parameters, which keeps the dynamic properties of the original system.

Numerical simulations are presented to illustrate our results with the theoretical analysis and show the effect of the control method.

The chaotic behavior of this model shows the relations of the different species, including the number population, birth rate and survival rate, whether they can survive in a balanced state or makes the population develop in disorder or chaos. This research provides some theoretical basis and may be helpful for the research in biology or ecology.

When the parameters of the system take a different set of values, what more complex dynamic behavior the system will have and whether the OGY method can realize the chaos control of the system in this period? These problems will be very interesting and need further research and discussion.

\section{Acknowledgement}

This work is supported by the High Level Scientific Research Project Cultivation Fund of Shandong Women's University.

\section{Ethics}

This article is original and contains unpublished material. The corresponding author confirms that all of the other authors have read and approved the manuscript and no ethical issues involved.

\section{References}

Alba-Pérez, J. and Macías-Díaz, J. E. (2019). Analysis of structure-preserving discrete models for predatorprey systems with anomalous diffusion. Mathematics, 7(12), 1172.

Alsedà, L., Vidiella, B., Solé, R., Lazaro, J. T. and Sardanyés, J. (2020). Dynamics in a time-discrete food-chain model with strong pressure on preys. Communications in Nonlinear Science and Numerical Simulation, 84, 105187.

Asheghi, R. (2014). Bifurcations and dynamics of a discrete predator-prey system. Journal of biological dynamics, 8(1), 161-186.

Bešo, E., Kalabušić, S., Mujić, N. and Pilav, E. (2020). Stability of a certain class of a host-parasitoid models with a spatial refuge effect. Journal of Biological Dynamics, 14(1), 1-31.

Bešo, E., Kalabušić, S., Mujić, N. and Pilav, E. (2019). Neimark-Sacker Bifurcation and Stability of a Certain Class of Host-Parasitoid Models with Host Refuge Effect. International Journal of Bifurcation and Chaos, 29(12), 1950169.

Costantino, R. F., Desharnais, R. A., Cushing, J. M. and Dennis, B. (1997). Chaotic dynamics in an insect population. Science, 275(5298), 389-391.

Dennis, B., Desharnais, R. A., Cushing, J. M., Henson, S. M. and Costantino, R. F. (2001). Estimating chaos and complex dynamics in an insect population. Ecological Monographs, 71(2), 277-303. 
Din, Q. (2017). Complexity and chaos control in a discrete-time prey-predator model. Communications in Nonlinear Science and Numerical Simulation, 49, 113-134.

Din, Q. (2018a). Bifurcation analysis and chaos control in discrete-time glycolysis models. Journal of Mathematical Chemistry, 56(3), 904-931.

Din, Q. (2018b). Controlling chaos in a discrete-time prey-predator model with Allee effects. International Journal of Dynamics and Control, 6(2), 858-872.

Din, Q., Donchev, T. and Kolev, D. (2018). Stability, bifurcation analysis and chaos control in chlorine dioxide-iodine-malonic acid reaction. MATCH Commun. Math. Comput. Chem, 79(3), 577-606.

Kapçak, S., Ufuktepe, Ü. and Elaydi, S. (2013). Stability and invariant manifolds of a generalized Beddington host-parasitoid model. Journal of biological dynamics, 7(1), 233-253.

Elsadany, A. E. A. (2012). Dynamical complexities in a discrete-time food chain. Computational Ecology and Software, 2(2), 124.

Feng, G., Pan, J. X., Yong, M. and Ji, Z. H. E. N. (2018). The dynamic property and chaos control for a twodegree-of-freedom vibro-impact system.
Guo, F., Xie, J. and Yuan, L. (2014a). Chaos Control of Lauwerier Mapping. Journal of Southwest Jiaotong University, 49(3), 525-529.

Guo, F., Xie, J. H. and Yue, Y. (2014b). Chaos control of Lozi mapping. In Applied Mechanics and Materials (Vol. 509, pp. 231-235). Trans Tech Publications Ltd.

Hastings, A. and Powell, T. (1991). Chaos in a three species food chain. Ecology, 72(3), 896-903.

Kacarev, L. and Parlitz, U. (1996). General approach for chaotic synchronization, with application to communication. Physical review letters, 74, 50285031 .

May, R. M. (1976). Simple mathematical models with very complicated dynamics. Nature, 261(5560), 459-467.

Ogata, K., (1990). Controlling Engineering. Prentice Hall, Englewood Cliffs, NJ.

Ott, E., Grebogi, C. and Yorke, J. A. (1990). Controlling chaos. Physical review letters, 64(11), 1196.

Pyragas, K. (1995). Control of chaos via extended delay feedback. Physics letters A, 206(5-6), 323-330.

Shinbrot, T., Grebogi, C., Yorke, J. A. and Ott, E. (1993). Using small perturbations to control chaos. Nature, 363(6428), 411-417.

Zhang, L. and Zhao, H. F. (2009). Periodic solutions of a three-species food chain model. Applied Mathematics E-Notes, 9, 47-54. 